\title{
Trapped modes in a two-layer fluid
}

\author{
By C. M. LINTON AND J. R. CADBY \\ Department of Mathematical Sciences, Loughborough University, Leicestershire LE11 3TU, UK
}

(Received 23 September 2002 and in revised form 21 November 2002)

Trapped-mode frequencies are computed for submerged horizontal circular cylinders in a two-layer fluid consisting of a finite layer bounded above by a free surface and below by an infinite layer of greater density. Two separate cases are examined. First we consider waves which propagate along the top of a single cylinder at frequencies below the cut-off for wave propagation away from the cylinder, and find modes where the motion is concentrated around the interface, modes which are concentrated around the free surface, and modes which involve significant motion of both the interface and the free surface. Secondly, we compute modes above the cut-off for a pair of submerged cylinders in the lower fluid.

\section{Introduction}

The existence of a trapped mode above a submerged, horizontal, circular cylinder in infinitely deep water was first established by Ursell (1951). These modes correspond to waves propagating along the top of the cylinder and decaying exponentially as one moves away from it. On the basis of the full linear theory of water waves, Ursell showed that the existence of trapped waves depended on the vanishing of a certain infinite determinant. He went on to prove that zeros of the determinant exist if the radius of the cylinder is sufficiently small compared to the depth of submergence of the cylinder. The restriction on cylinder size was removed by Jones (1953). McIver \& Evans (1985) subsequently computed the frequencies at which trapped modes occur by evaluating the determinant numerically.

Kuznetsov (1993) studied the existence of trapped modes above a cylinder of constant cross-section in the (infinite) lower layer of a two-layer fluid. Using a perturbation method Kuznetsov showed that, provided the density difference between the two layers is small, there are two sets of frequencies at which trapped modes might exist: one set corresponding to disturbances on the free surface which are similar to those for the single-layer fluid case, and the other set corresponding to trapped modes on the interface between the two layers. In $\$ 2$ we use the theory developed in Linton \& McIver (1995) and Linton \& Cadby (2002, hereafter referred to as LC) to compute the frequencies at which trapped modes occur for the case of a submerged horizontal circular cylinder in either the upper or lower layer of a two-layer fluid. The calculations presented in this paper suggest that only the set of interfacial modes predicted by Kuznetsov (1993) exist; the frequencies at which the free-surface modes would exist are such that waves propagate away from the obstacle along the interface. In practical terms this means that if a mode corresponding to a trapped mode in a single-layer fluid is excited in a two-layer fluid, the energy in this mode will leak away along the interface, no matter how small the density ratio between the layers is. 
All the modes discussed above occur at frequencies below a cut-off. Above this cutoff it is possible to formulate a scattering problem corresponding to the interaction of an obliquely incident wave with the cylinder, but below it no waves can propagate into the far field.

However, for the two-dimensional water-wave problem, McIver (1996) proved that trapped modes could exist in the absence of a cut-off near to pairs of surface-piercing structures, and more recently McIver (2000) has extended this to pairs of submerged structures. In each case the actual shape of the structures is not known in simple form and must be computed numerically. To date the only simple shapes for which this type of trapped mode have been computed are pairs of angled barriers (Linton \& Kuznetsov 1997), two pairs of vertical barriers (Kuznetsov, McIver \& Linton 2001), and pairs of submerged ellipses (Porter 2002). In each case the geometry consists of a pair of obstacles, each of which exhibits the phenomenon of total reflection at certain discrete frequencies. The physical justification for the link between these phenomena is that if the structures are sufficiently far apart so that one does not interfere with the other, waves can travel back and forth between them, with no energy being transmitted past the structures into the far field.

The ideas of McIver (1996) were extended to the oblique wave case in Kuznetsov et al. (1998) and Motygin (1999). They showed that pairs of surface-piercing structures could trap waves at frequencies above the cut-off, though the surfaces themselves had to be computed numerically. In LC frequencies were found at which obliquely incident interfacial waves are totally reflected by a submerged horizontal circular cylinder in a two-layer fluid and it is thus natural to ask if trapped modes can be found in the presence of pairs of such cylinders. The answer would appear to be yes, and the relevant calculations are described in $\S 3$.

\section{Single cylinder}

Cartesian coordinates are chosen such that the $(x, y)$-plane coincides with the undisturbed interface between the two fluids. The $z$-axis points vertically upwards with $z=0$ as the interface and $z=d>0$ as the free surface. Under the usual assumptions of linear water-wave theory we can define a velocity potential in the form

$$
\Phi(x, y, z, t)=\operatorname{Re}\left\{\phi(x, z) \mathrm{e}^{\mathrm{i} l y} \mathrm{e}^{-\mathrm{i} \omega t}\right\}
$$

and since $\Phi$ is harmonic, $\phi$ must satisfy the modified Helmholtz equation

$$
\left(\nabla_{x z}^{2}-l^{2}\right) \phi=0
$$

The upper fluid, $0<z<d$, will be referred to as region $I$, whilst the lower fluid, $z<0$, will be referred to as region $I I$. The potential in the upper fluid (of density $\rho^{I}$ ) will be denoted by $\phi^{I}$ and that in the lower fluid (of density $\rho^{I I}>\rho^{I}$ ) by $\phi^{I I}$. If we define $\rho=\rho^{I} / \rho^{I I}(<1)$ then the linearized boundary conditions on the interface and free surface are

$$
\begin{array}{ll}
\phi_{z}^{I}=\phi_{z}^{I I} & \text { on } z=0 \\
\rho\left(\phi_{z}^{I}-K \phi^{I}\right)=\phi_{z}^{I I}-K \phi^{I I} & \text { on } z=0, \\
\phi_{z}^{I}=K \phi^{I} & \text { on } z=d,
\end{array}
$$

where $K=\omega^{2} / g, g$ being the acceleration due to gravity. The boundary conditions 
(2.3) and (2.4) represent the continuity of normal velocity and pressure at the interface respectively.

Within this framework progressive waves take the form (up to an arbitrary multiplicative constant)

$$
\begin{gathered}
\phi^{I}=\mathrm{e}^{ \pm \mathrm{i} x \sqrt{u^{2}-l^{2}}}\left((u+K) \mathrm{e}^{u(z-d)}+(u-K) \mathrm{e}^{-u(z-d)}\right), \\
\phi^{I I}=\mathrm{e}^{ \pm \mathrm{i} x \sqrt{u^{2}-l^{2}}} \mathrm{e}^{u z}\left((u+K) \mathrm{e}^{-u d}-(u-K) \mathrm{e}^{u d}\right),
\end{gathered}
$$

where either $u=K$ or $u$ satisfies the relation

$$
K\left(\sigma+\mathrm{e}^{-2 k d}\right)=k\left(1-\mathrm{e}^{-2 k d}\right),
$$

in which $\sigma=(1+\rho) /(1-\rho)$. This equation has exactly one positive root $k$, which is always greater than $K$.

For trapped modes we require

$$
\phi,|\nabla \phi| \rightarrow 0 \quad \text { as } \quad|x| \rightarrow \infty
$$

and so we restrict $l$ to be in the range $l>k>K$. This ensures that there are no waves propagating to infinity on either the free surface or the interface. The far-field behaviour can be then shown to be of the form

$$
\begin{aligned}
& \phi^{I} \sim A^{ \pm} \mathrm{e}^{\mp \beta x} \mathrm{e}^{K z}+B^{ \pm} \mathrm{e}^{\mp b x} g(z), \\
& \phi^{I I} \sim A^{ \pm} \mathrm{e}^{\mp \beta x} \mathrm{e}^{K z}+B^{ \pm} \mathrm{e}^{\mp b x} \mathrm{e}^{k z},
\end{aligned}
$$

as $x \rightarrow \pm \infty$, where $\beta=\sqrt{l^{2}-K^{2}}, b=\sqrt{l^{2}-k^{2}}$, and

$$
g(z)=\frac{K \sigma-k}{K(\sigma-1)} \mathrm{e}^{k z}+\frac{K-k}{K(\sigma-1)} \mathrm{e}^{-k z} .
$$

Cylinder in the lower fluid layer

We seek trapped modes symmetric about the line $x=0$ and write the velocity potential as

$$
\phi=\sum_{m=0}^{\infty} \alpha_{m} \phi_{m}^{s},
$$

where $\phi_{m}^{s}$ are the multipoles defined in equations (3.2) and (3.3) of LC. When expanded about $z=f(<0)$ we have, with $x=r \sin \theta, z=f-r \cos \theta$,

$$
\phi_{m}^{s}=K_{m}(l r) \cos m \theta+\sum_{n=0}^{\infty} A_{m n}^{s} I_{n}(l r) \cos n \theta,
$$

where, with $\epsilon_{0}=1$ and $\epsilon_{n}=2, n=1,2, \ldots$, and $v=l \cosh u$,

$$
\begin{gathered}
A_{m n}^{s}=(-1)^{m+n} \epsilon_{n} \int_{0}^{\infty} \cosh m u \cosh n u \mathrm{e}^{2 v f} C(v) \mathrm{d} u, \\
C(v)=(v+K)\left[(v+K \sigma) \mathrm{e}^{-2 v d}-v+K\right] /[(v-K) h(v)], \\
h(v)=(v+K) \mathrm{e}^{-2 v d}-v+K \sigma .
\end{gathered}
$$

We note that since $v>l>k>K$ there will be no singularities of the integrand on the real axis. Applying the body boundary condition, $\partial \phi / \partial r=0$ on $r=a$, we obtain 
an infinite system of linear equations for the unknowns $\alpha_{m}$, which is

$$
\alpha_{n}+\frac{I_{n}^{\prime}(l a)}{K_{n}^{\prime}(l a)} \sum_{m=0}^{\infty} \alpha_{m} A_{m n}^{s}=0 .
$$

For a fixed geometrical configuration, we find the relationship that must exist between $k$ and $l$ for trapped modes to exist by fixing one parameter, say $l a$, and varying the other, say $k a$, to find the zeros of the suitably truncated determinant. The results presented below were obtained to two decimal places and for some parameter values a $32 \times 32$ system was required.

In order to appreciate the nature of the trapped modes in two-layer fluids it will be helpful to look at the profiles of the free surface and interface. The free-surface and interfacial elevations, denoted by $H_{d}$ and $H_{0}$ respectively, can be written in the form $H=\operatorname{Re}\left\{\eta(x) \mathrm{e}^{\mathrm{i} l y} \mathrm{e}^{-\mathrm{i} \omega t}\right\}$ and then

$$
\eta_{d}(x)=\frac{\mathrm{i}}{\omega} \phi_{z}^{I}(x, d), \quad \eta_{0}(x)=\frac{\mathrm{i}}{\omega} \phi_{z}^{I}(x, 0),
$$

where, from (2.13) and LC, equation (3.2),

$$
\begin{array}{r}
\phi_{z}^{I}(x, d)=\sum_{m=0}^{\infty} \frac{4(-1)^{m} K^{2} \alpha_{m}}{1-\rho} \int_{0}^{\infty} v \cosh m u \cos (l x \sinh u) \frac{\mathrm{e}^{v(f-d)}}{(v-K) h(v)} \mathrm{d} u, \quad(2.20) \\
\phi_{z}^{I}(x, 0)=\sum_{m=0}^{\infty} \frac{2(-1)^{m} K \alpha_{m}}{1-\rho} \int_{0}^{\infty} v \cosh m u \cos (l x \sinh u) \mathrm{e}^{v f} \frac{\left[(v+K) \mathrm{e}^{-2 v d}-v+K\right]}{(v-K) h(v)} \mathrm{d} u .
\end{array}
$$

The non-dimensionalized normalized maximum displacements of the free surface and interface from their mean positions, denoted $Y_{d}$ and $Y_{0}$, are written

$$
Y_{d}(X)=\phi_{z}^{I}(X, d / a) / \bar{\eta}, \quad Y_{0}(X)=\phi_{z}^{I}(X, 0) / \bar{\eta},
$$

where $\bar{\eta}=\max \left(\left|\phi_{z}^{I}(0, d / a)\right|,\left|\phi_{z}^{I}(0,0)\right|\right)$ and $X=x / a$. This has the effect of scaling the amplitudes so that the maximum deviation at $x=0$ is unity.

Figures 1-3 show the results obtained for trapped modes above a circular horizontal cylinder situated in the lower fluid of a two-layer fluid. In all the figures the submergence, $f / a$, of the cylinder is -1.01 which is very close to the interface. As expected, the results for the infinite-depth single-layer fluid problem are recovered when $\rho=0$ (with the interface now playing the role of the free surface).

Figure 1 shows the trapped-mode frequencies for a density ratio of $\rho=0.5$. The different curves correspond to four different depths of the upper fluid layer: $d / a=0.5$, $1,1.5$ and 2 , and for each value of $d / a$ there are two curves. The existence of trapped modes requires $l>k$ so that $l=k$ (thin dashed line) is an upper bound for these curves. The curves are similar to those obtained for a single-layer fluid by McIver $\&$ Evans (1985). We notice that as we increase the depth of the upper fluid layer these trapped mode frequencies decrease. By fixing $d / a$ and $\rho$ and varying the submergence we can find different numbers of modes. As $f / a$ approaches -1 we see a 'fanning out' of the curves as higher modes appear, just like in the single-layer case.

Figure 2 shows the trapped-mode frequencies plotted against $\rho$ when $l a=2$. There are four sets of results corresponding to different depths of the upper fluid layer. These are $d / a=0.8,1.0,1.2$ and 2.0 and for each of these values there are two curves showing the first and second modes. In the limit as $\rho \rightarrow 1$ we see from figure $2(b)$ 


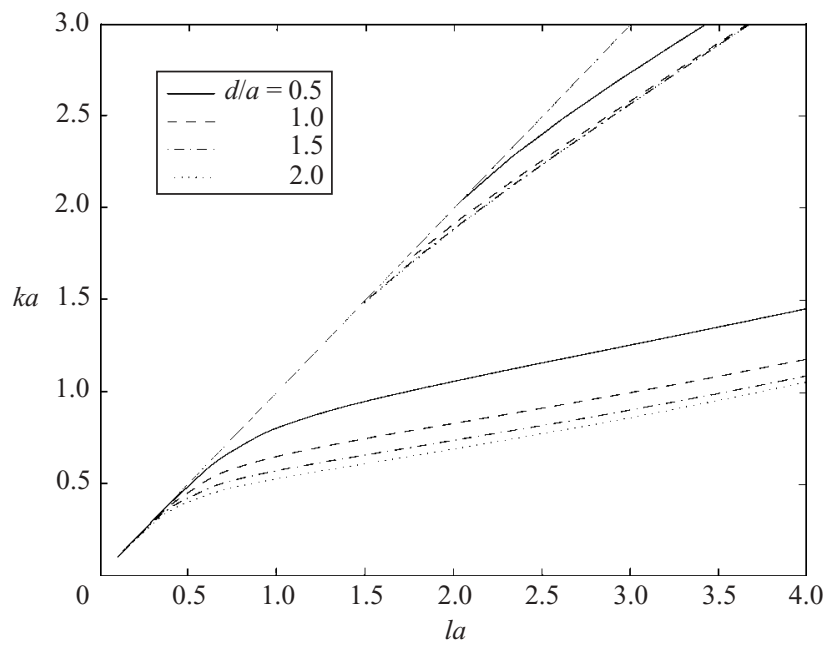

FIGURE 1. Trapped-mode frequencies for a cylinder in the lower fluid layer; $\rho=0.5$ and $f / a=-1.01$.

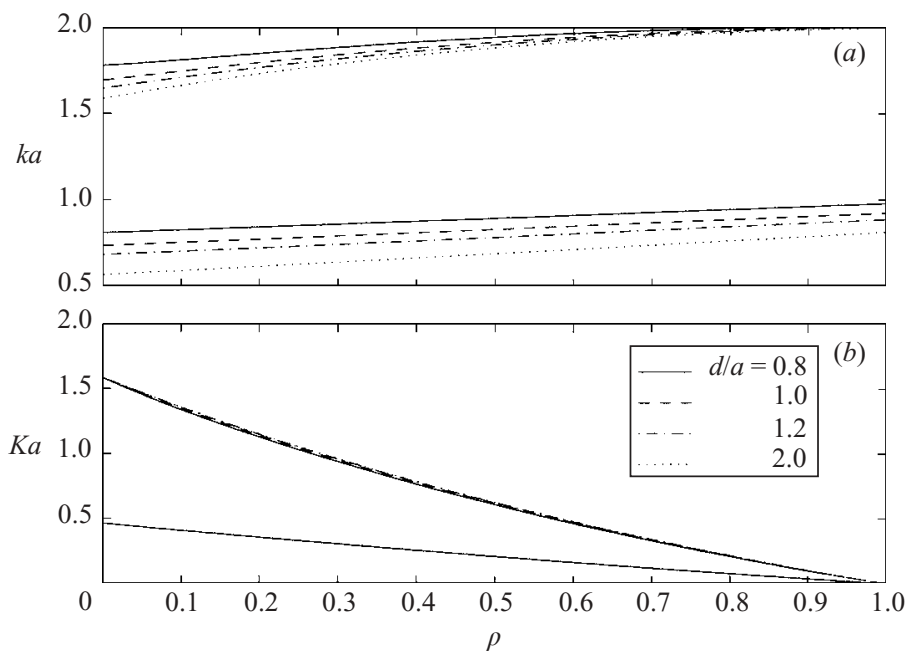

FIGURE 2. Trapped-mode frequencies for a cylinder in the lower fluid layer; $l a=2$ and $f / a=-1.01$.

that the frequency $K a$ of the trapped modes tends to zero while the wavenumber $k a$, shown in figure $2(a)$, tends to some limit for each mode. One might expect that as $\rho \rightarrow 1$ (in which case the upper and lower fluids have equal density) we would recover the single-layer fluid results where the submergence of the cylinder is $f / a-d / a$. However, this is not the case for reasons which are discussed at the end of this section.

In figure 3 we show the free-surface and interfacial profiles $Y_{d}$ and $Y_{0}$, respectively, of the trapped modes that exist when $l a=2$ and $d / a=1$. The different curves correspond to six different values of the density ratio: $\rho=0,0.2,0.4,0.6,0.8$ and 0.999. The left-hand plots are for the first mode, with no crossings of $Y_{0}=0$ or $Y_{d}=0$, corresponding to the lower frequencies in figure 2 whilst the right-hand plots are for 

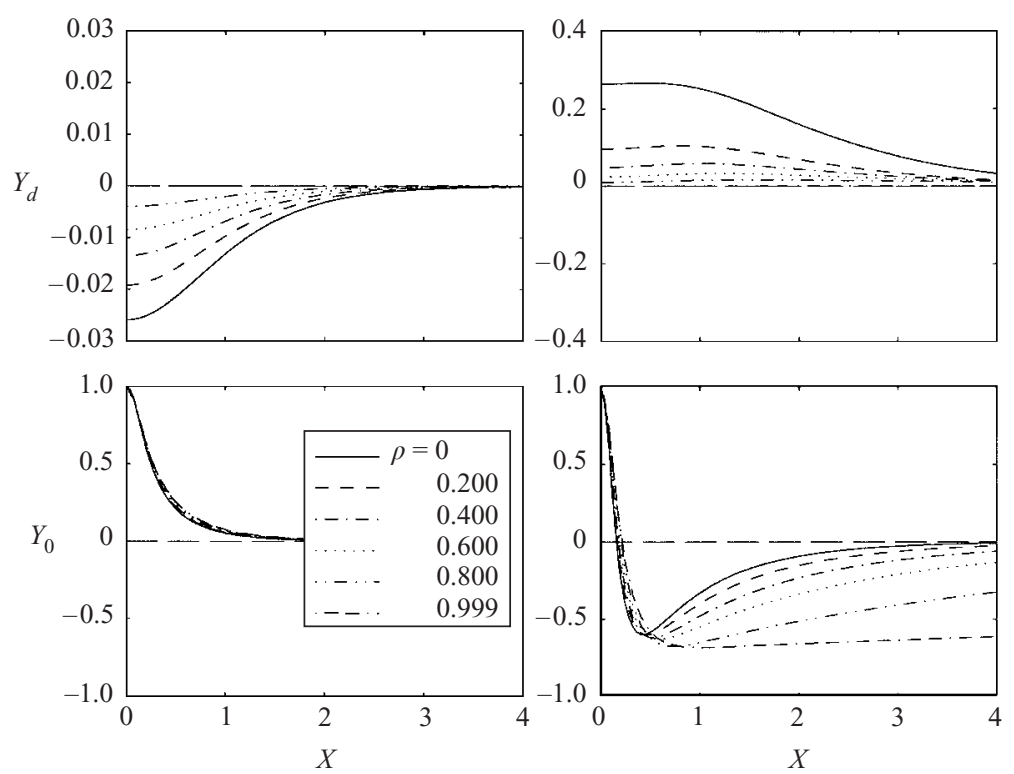

FIGURE 3. The free-surface and interfacial profiles of trapped modes for a cylinder in the lower fluid layer; $l a=2, f / a=-1.01$ and $d / a=1.0$.

the second mode, with one zero crossing on the interface, corresponding to the higher frequencies. For both modes the motion is concentrated about the interface. The free surface and interface are out of phase for the first mode whereas they are in phase for the second. We can see that the amplitude of the free surface becomes very small as $\rho \rightarrow 1$ for both modes. The shape of the interface profile for the first mode barely changes over the range of values of $\rho$. For the second mode, however, the disturbance on the interface projects further into the fluid as $\rho \rightarrow 1$. This is explained by the behaviour of $k$ in the limit as $\rho \rightarrow 1$. In the far-field form of the potential we have an $\exp \left(-\sqrt{l^{2}-k^{2}}|x|\right)$ term. From figure 2, we see that as $\rho \rightarrow 1$ the wavenumber of the second mode $k a \rightarrow 2=l a$. Thus the rate of decay of the exponential term decreases as $\rho$ becomes closer to unity and in the limit there is no trapped mode.

\section{Cylinder in the upper layer}

For the case of a cylinder in the upper fluid layer $(f>0)$ the velocity potential can be expanded using (2.13), but we now use the multipoles defined in equations (4.1) and (4.2) of LC. When expanded about $r=0$ we now have

$$
\phi_{m}^{s}=K_{m}(l r) \cos m \theta+\sum_{n=0}^{\infty} B_{m n}^{s} I_{n}(l r) \cos n \theta,
$$

where

$$
\begin{gathered}
B_{m n}^{s}=\epsilon_{n} \int_{0}^{\infty} \cosh m u \cosh n u\left[(-1)^{n} A(v) \mathrm{e}^{v f}+B(v) \mathrm{e}^{-v f}\right] \mathrm{d} u, \\
A(v)=\frac{(v+K) \mathrm{e}^{-2 v d}\left[(-1)^{m+1}(v-K \sigma) \mathrm{e}^{v f}-(v-K) \mathrm{e}^{-v f}\right]}{(v-K)\left((v+K) \mathrm{e}^{-2 v d}-v+K \sigma\right)}, \\
B(v)=\frac{(-1)^{m+1}(v+K) \mathrm{e}^{v(f-2 d)}-(v-K) \mathrm{e}^{-v f}}{(v+K) \mathrm{e}^{-2 v d}-v+K \sigma} .
\end{gathered}
$$




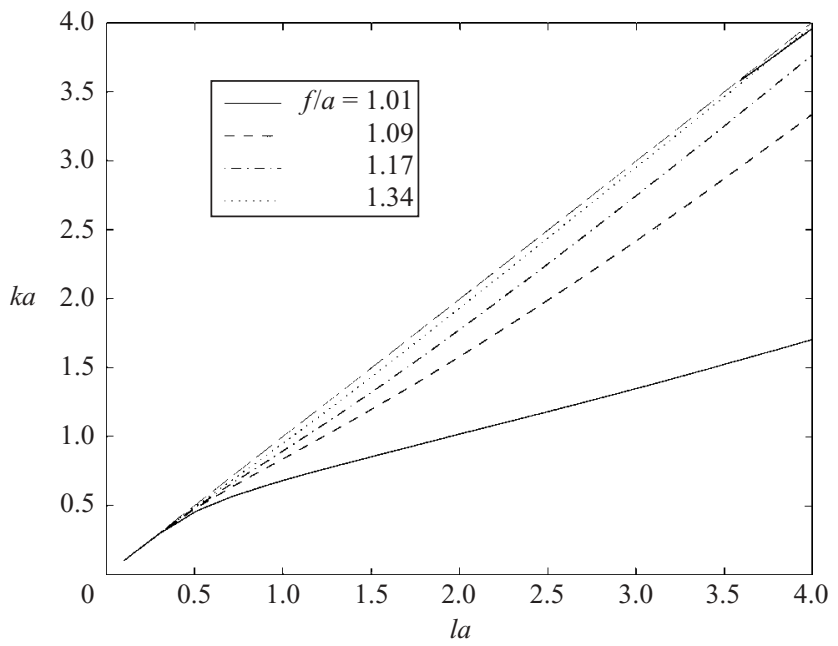

FIGURE 4. Trapped-mode frequencies for a cylinder in the upper fluid layer; $d / a=3$ and $\rho=0.5$.

Again, the integrand has no singularities on the real axis. By applying the body boundary condition, $\partial \phi / \partial r=0$ on $r=a$, we obtain the same system of equations (2.18) for $\alpha_{m}$ as before, except $A_{m n}^{s}$ is replaced by $B_{m n}^{s}$.

The free-surface and interfacial elevations are again given by (2.22) but we use the following forms for $\phi_{z}^{I}$ :

$$
\begin{aligned}
& \phi_{z}^{I}(x, d)=\sum_{m=0}^{\infty} 2 K \alpha_{m} \int_{0}^{\infty} v \cosh m u \cos (l x \sinh u) \\
& \times \frac{\mathrm{e}^{-v d}\left[(-1)^{m}(K \sigma-v) \mathrm{e}^{v f}+(v-K) \mathrm{e}^{-v f}\right]}{(v-K) h(v)} \mathrm{d} u, \\
& \phi_{z}^{I}(x, 0)=\sum_{m=0}^{\infty}(\sigma-1) K \alpha_{m} \int_{0}^{\infty} v \cosh m u \cos (l x \sinh u) \\
& \times \frac{(-1)^{m}(v+K) \mathrm{e}^{v(f-2 d)}+(v-K) \mathrm{e}^{-v f}}{(v-K) h(v)} \mathrm{d} u,
\end{aligned}
$$

Figure 4 shows dispersion curves for the case $d / a=3, \rho=0.5$. There are four curves corresponding to different submergence depths of the cylinder: $f / a=1.01$, $1.09,1.17$ and 1.34. The upper bound for trapped modes $k=l$ is included, shown by the thin dashed line. As the cylinder approaches the interface, $f / a \rightarrow 1$, the curves fold out from the upper bound $k=l$. Similar effects are observed when the cylinder approaches the free surface.

In figure 5 we show the trapped-mode frequencies plotted against the density ratio when $l a=2$ and $d / a=2.1$. The results presented are for four values of submergence: $f / a=1.05,1.06,1.07$ and 1.08. For each value of submergence there are two curves. When $\rho=0$ we recover the single-layer finite-depth fluid results (see figures 10 and 11 below) for depth $d / a$ and cylinder submergence $f / a-d / a$. The first modes, the lower of each pair of curves, correspond to lines of constant $K a$ which are solutions to the single-layer fluid problem. These first modes appear to cross the second modes, the higher curves, but with closer inspection at these points, see figure 6 , we observe near 


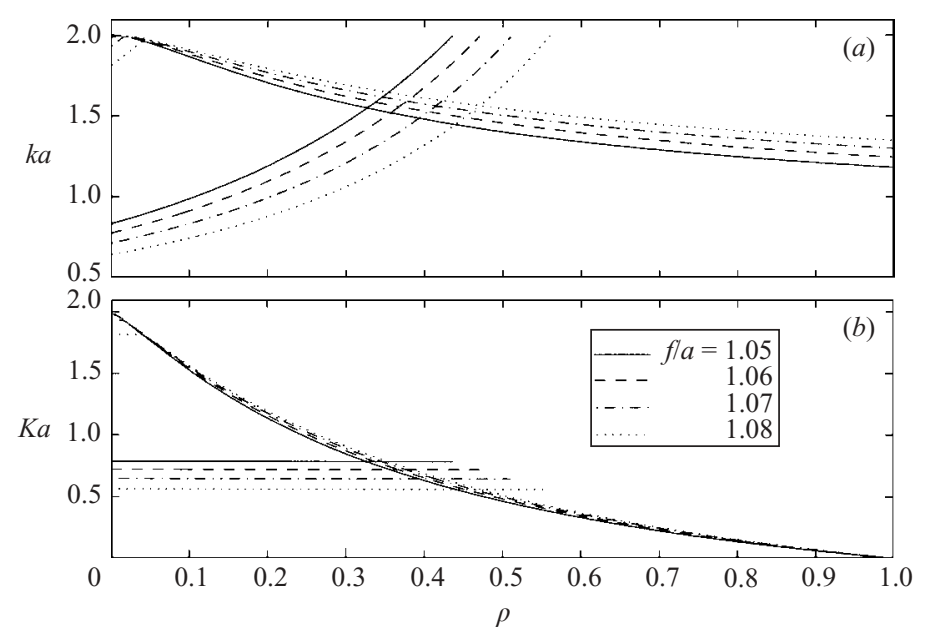

FIGURE 5. Trapped-mode frequencies for a cylinder in the upper fluid layer; $l a=2$ and $d / a=2.1$.

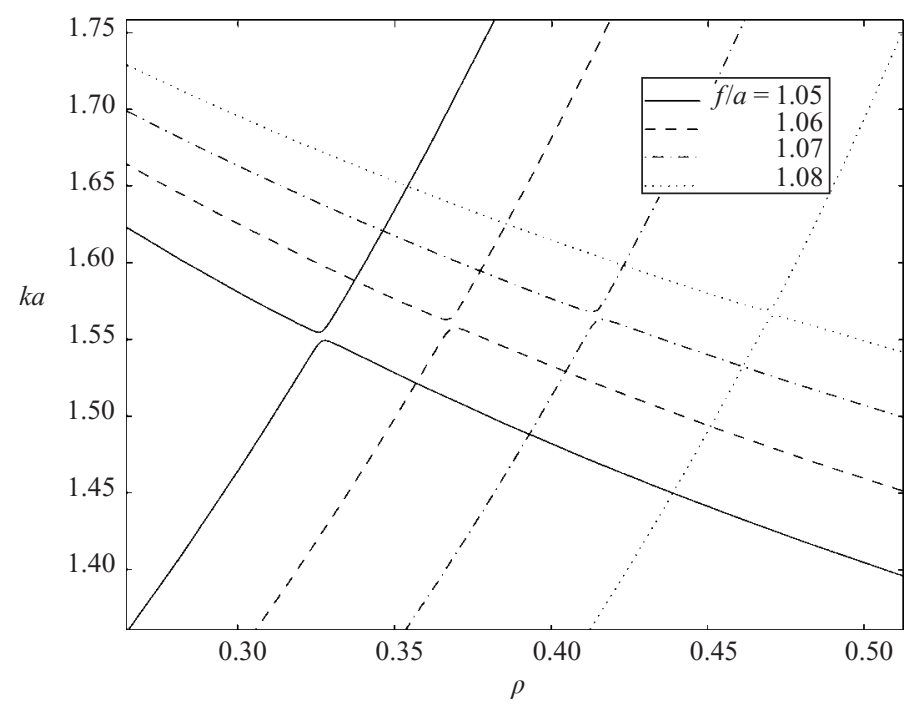

FIGURE 6. Trapped-mode frequencies for a cylinder in the upper fluid layer, close-up of near crossing; $l a=2$ and $d / a=2.1$.

crossing. These near crossings, or diabolical points as they are described by Berry \& Wilkinson (1984), have been observed in other trapped-mode problems, see for example Linton \& McIver (1998). Thus, for increasing $\rho$ the first and second modes come very close to each other at some point, after which the second mode terminates at $k a=2=l a$ and the first tends to some value as $\rho \rightarrow 1$ (see discussion on this limit below). For $f / a=1.08$ (the dotted curves) there is in fact a third mode for small $\rho$. This third mode has a near crossing with the second mode at around $\rho \approx 0.045$ and then terminates when it reaches $k a=2$. For cylinders closer to the free surface more modes will appear and there will be more near crossings.

Figures 7-9 show the free-surface and interfacial profiles for $f / a=1.05, d / a=2.1$ and $l a=2$, and increasing density. The left-hand plots in figures $7-9$ are the profiles 

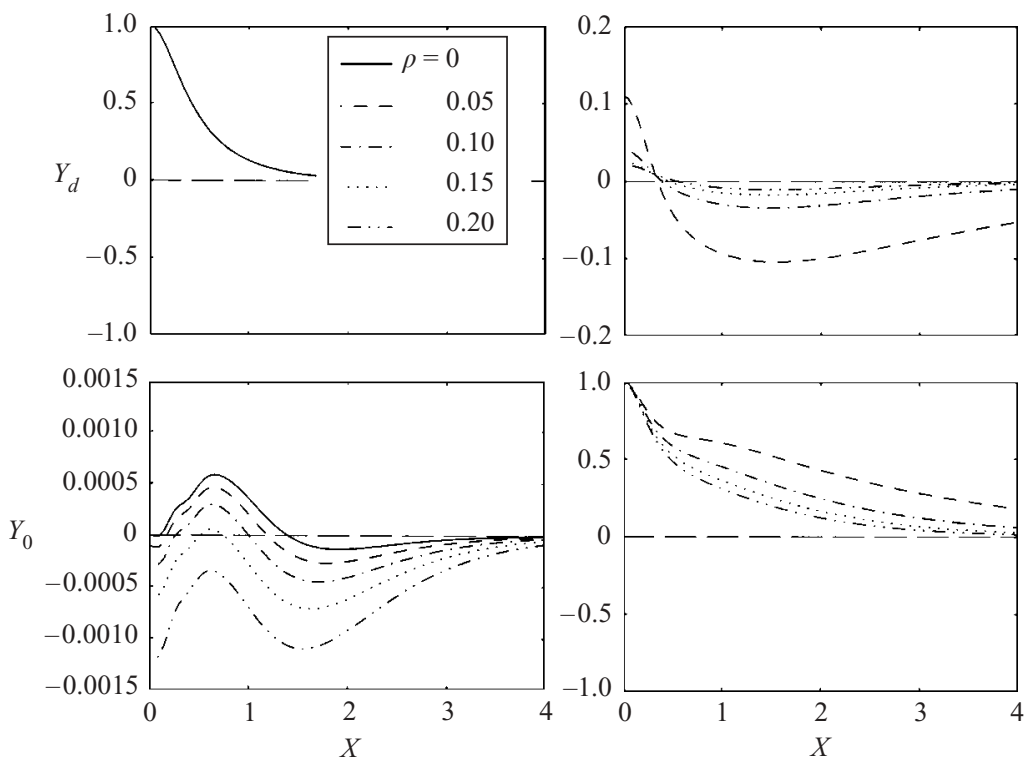

FIGURE 7. Free-surface and interfacial profiles for a cylinder in the upper fluid layer; $f / a=1.05, d / a=2.1$ and $l a=2$.
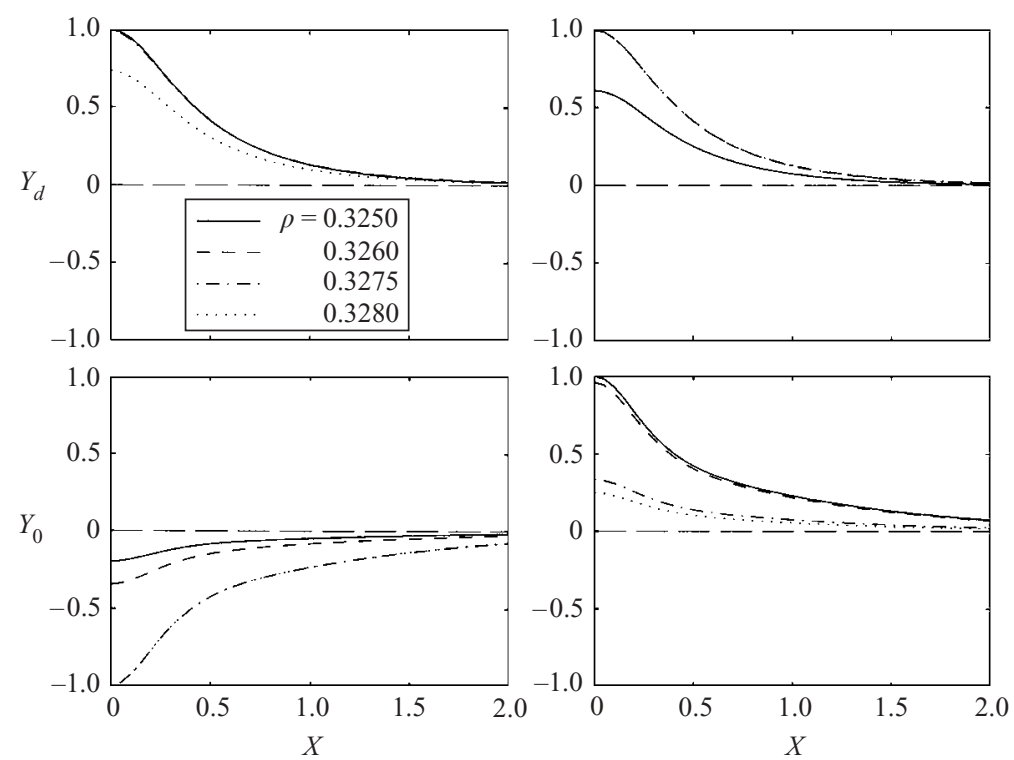

FIGURE 8. Free-surface and interfacial profiles for a cylinder in the upper fluid layer; $f / a=1.05, d / a=2.1, l a=2$.

corresponding to the lower frequencies of the solid curve shown in figure 5, whereas the right-hand plots are the profiles corresponding to the higher frequencies. The upper graphs are the free-surface profiles while the lower graphs are the interfacial profiles. For all values of $\rho$ used, the free-surface and interfacial profiles are in phase for the second modes and out of phase for the first modes. For low density ratios, shown in figure 7, the motion for the first mode is concentrated about the free surface 

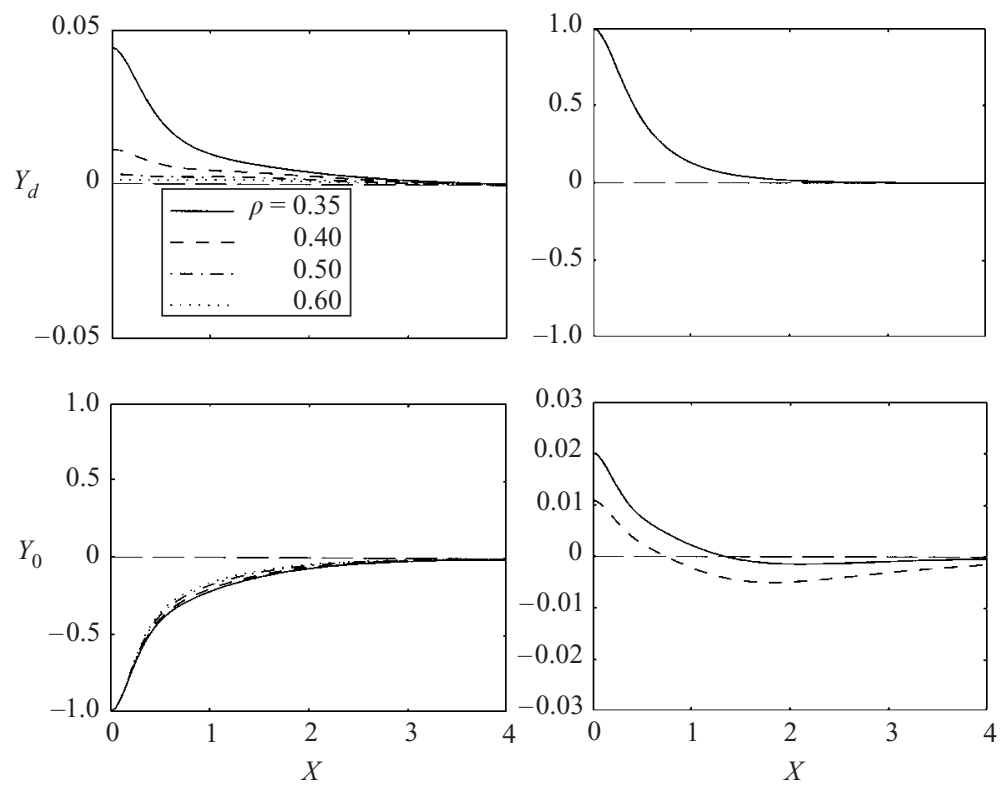

FIGURE 9. Free-surface and interfacial profiles for a cylinder in the upper fluid layer; $f / a=1.05, d / a=2.1$ and $l a=2$.

and for the second mode the motion is concentrated about the interface. As the density ratio is increased the motion for the first mode (left-hand graphs) is transferred from the free surface to the interface. At a density ratio of $\rho \approx 0.3275$ the free-surface and interfacial amplitudes for the first mode at $X=0$ are equal. For the second mode we have a similar effect where the motion transfers from the interface to the free surface at $\rho \approx 0.326$. We note that both these changes take place in the region of the near crossing of the modes shown by the solid curves in figure 6 . If we follow the first (second) mode with increasing $\rho$ we begin with a free-surface (interface) trapped mode which later becomes an interface (free-surface) trapped mode. About the nearcrossing point trapped modes can exist on both the free surface and interface for both modes. It seems likely that if there were more modes with near-crossing points then these points would have similar properties. The second mode stops at $\rho \approx 0.435$ and approaching this value the motion remains on the free surface with very little happening at the interface. As $\rho$ increases towards unity the motion of the first mode becomes confined to the interface and the free-surface elevation becomes very small.

The limit $\rho \rightarrow 0$ corresponds to $\rho^{I I} \rightarrow \infty$. The dispersion relation reduces to $K=k \tanh (k d)$ and the multipoles become those for a single-layer finite-depth fluid. Thus by letting $\rho \rightarrow 0$ we obtain results for trapped modes above a cylinder in a finite-depth homogeneous fluid. Such modes do not appear to have been computed before. The results presented below were computed to three decimal places for which in some cases a $40 \times 40$ system was required. Generally, higher frequencies required more terms than lower frequencies.

In figure 10 the different curves correspond to different submergence depths, $f / a$, of the cylinder: $-1.01,-1.05,-1.1$, and -1.5 , where the depth, $h / a$, of the fluid is 3 . For the submergence $f / a=-1.5$ we see there is just a single mode and that the curve lies close to the upper bound. As the cylinder approaches the free surface, $|f / a| \rightarrow 1$, the frequency of the first mode decreases and as we come closer still a 


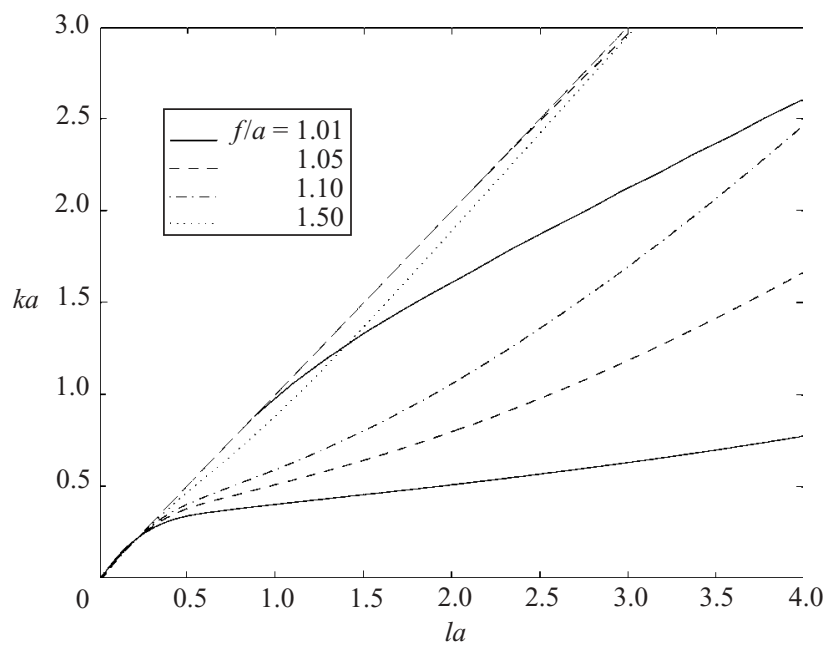

FIGURE 10. Trapped-mode frequencies in a finite-depth single-layer fluid; $h / a=3$.

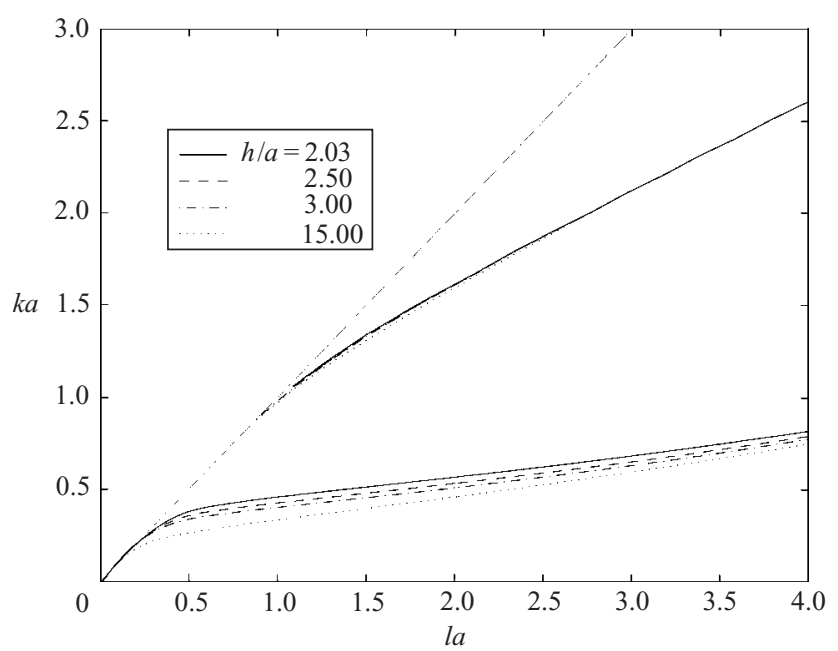

FIGURE 11. Trapped-mode frequencies in a finite-depth single-layer fluid; $f / a=-1.01$.

second mode appears. For $f / a=-1.05$ a second mode exists for $2.1 \leqslant l a \leqslant 19.4$. When $f / a=-1.01$ there is a third mode appearing at $k a \approx 6$. These results are very similar to those found in McIver \& Evans (1985), and it would seem likely that the number of possible modes increases without bound as the top of the cylinder approaches the free surface. In figure 11 the curves correspond to different depths, $h / a$, of the fluid: $2.03,2.5,3$ and 15 , where the submergence of the cylinder has the value $f / a=-1.01$. We can see that the frequencies of the trapped modes are increased by the presence of the finite depth, the first mode being affected more that the second.

\section{Limit as $\rho \rightarrow 1$}

We might expect to recover the infinite-depth single-layer-fluid trapped-mode results from the two-layer analysis by letting $\rho \rightarrow 1$. However, as the results presented above 
indicate, this is not the case. In the limit $\rho \rightarrow 1$, we find that $K / k \rightarrow 0$ and so if $l(>k)$ is fixed then $K$ must tend to zero. Figures 2 and 5 show the trapped-mode frequencies versus the density ratio for a cylinder in the lower and upper fluid, respectively. For each set of parameter values there are two curves corresponding to two modes which are displayed in the graphs. We have already discussed the behaviour of the second mode as $\rho \rightarrow 1$ in the results above. For a cylinder in either fluid layer, the lower mode tends to some value which is a trapped mode but certainly does not correspond to one of a single-layer fluid because $K a \rightarrow 0$. To explain what is happening we shall consider the boundary conditions in the limit as $\rho \rightarrow 1$ and $K \rightarrow 0$ simultaneously.

We introduce small parameters $\varepsilon$ and $\delta$ and define

$$
K=\varepsilon, \quad \rho=1-\delta, \quad K^{\prime}=\frac{K}{1-\rho}=\frac{\varepsilon}{\delta}=O(1),
$$

such that when $\rho \rightarrow 1$ along one of the lower curves in figure 2 or 5 , which is for fixed $l$, we have $\varepsilon \rightarrow 0$ and $\delta \rightarrow 0$. In this limit, the free-surface condition becomes

$$
\phi_{z}^{I}=0 \quad \text { on } \quad z=d,
$$

the continuity of vertical velocity at the interface remains the same, $\phi_{z}^{I}=\phi_{z}^{I I}$ on $z=0$, and the continuity of pressure on the interface becomes

$$
K^{\prime} \phi^{I I}-\phi_{z}^{I I}=K^{\prime} \phi^{I} \text {. }
$$

The dispersion relation will have only one solution, $k$, which must satisfy

$$
2 K^{\prime}=k\left(1-\mathrm{e}^{-2 k d}\right) \text {. }
$$

In the absence of any bodies, oblique waves propagating in the fluid take the form

$$
\begin{aligned}
& \phi^{I}=A \mathrm{e}^{ \pm \mathrm{i} b x} \cosh k(z-d), \\
& \phi^{I I}=-A \mathrm{e}^{ \pm \mathrm{i} b x} \mathrm{e}^{k z} \sinh k d,
\end{aligned}
$$

where $b=\sqrt{k^{2}-l^{2}}$. For trapped modes we require $l>k$ so that the motion decays as $|x| \rightarrow \infty$. Thus, in the limit $\rho \rightarrow 1$ for fixed $l$ we obtain a boundary-value problem in terms of the new spectral parameter $K^{\prime}$. It is simple to find the forms of the multipoles for this problem and compute the trapped-mode frequencies. The results match those found in the limit of figures 2 and 5 and so we have related the limits of the trapped-mode curves in these figures to the trapped modes for the limiting problem defined above.

For trapped modes in a single-layer fluid we have the condition $K<l$ and for a two-layer fluid we have $K<k<l$. In the limit as $\rho \rightarrow 1$ we have $K / k \rightarrow 0$ by the dispersion relation. As we have shown above, in the limit $\rho \rightarrow 1$ when $l$ is fixed we have $K \rightarrow 0$. If we fix $K$ and let $\rho \rightarrow 1$ then we have $k \rightarrow \infty$ and hence $l \rightarrow \infty$. It is thus not possible to recover the single-layer-fluid results in the limit $\rho \rightarrow 1$.

\section{Two cylinders}

Here we shall consider the case of a pair of identical horizontal cylinders arranged symmetrically about a vertical line in a two-layer fluid. Only oscillations symmetric about this line are considered so that the geometry is equivalent to a horizontal cylinder next to a vertical wall. We then look for embedded trapped modes, modes for which $l$ is not greater than $k$, such that the wave motion is confined to the area between the cylinders. The frequencies at which zeros of transmission occur for a 


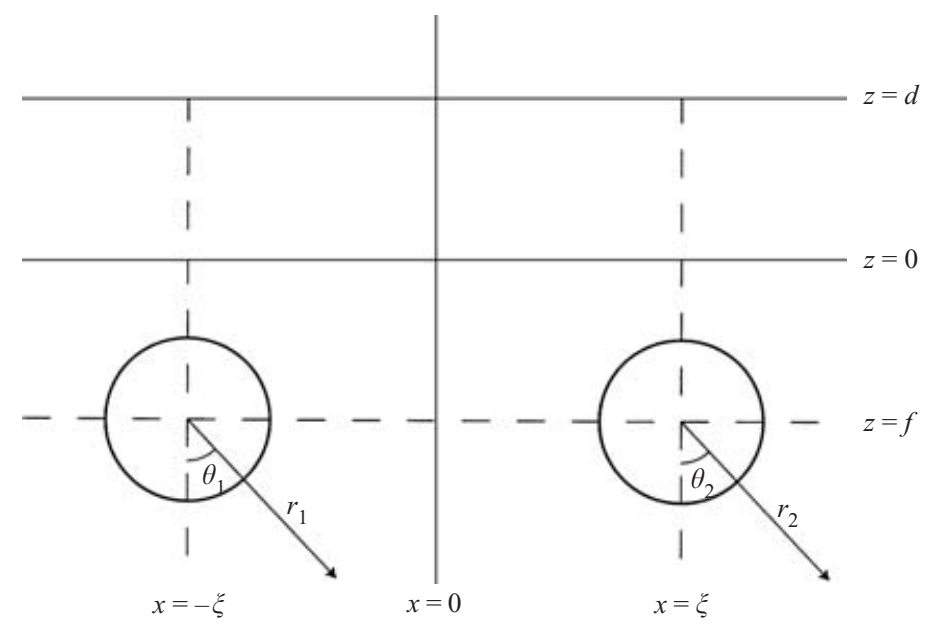

Figure 12. Definition sketch.

single cylinder provide a clue as to where one might start searching for trapped-mode frequencies. Physically, if the cylinders in our geometry are far enough apart it is reasonable to suggest that the presence of one cylinder will not influence what occurs at the other. Thus a wave which exists between the cylinders at a frequency for which zero transmission occurs might be totally reflected by one cylinder and then travel to the other cylinder and again be totally reflected, thus leading to a trapped oscillation. The problem will be formulated as in Linton \& Kuznetsov (1997), leading to the need to calculate the zeros of a complex determinant. Complications arise from this method which we will overcome by using the alternative formulation given by Evans \& Porter (1998).

Two circular horizontal cylinders of radius $a$ are centred on $(x, z)=(-\xi, f)$ and $(\xi, f)$ as in figure 12 and we will consider only modes symmetric about $x=0$, so that

$$
\frac{\partial \phi}{\partial x}=0 \quad \text { on } \quad x=0 .
$$

Here, for simplicity, we shall only consider the case of a cylinder in the lower fluid layer $(f<0)$ and work entirely with $\phi^{I I}$ (the superscript $I I$ will henceforth be omitted). We use polar coordinates $\left(r_{1}, \theta_{1}\right)$ and $\left(r_{2}, \theta_{2}\right)$ centred about $(-\xi, f)$ and $(\xi, f)$, respectively, such that

$$
\begin{gathered}
x=-\xi+r_{1} \sin \theta_{1}=\xi+r_{2} \sin \theta_{2}, \\
z=f-r_{1} \cos \theta_{1}=f-r_{2} \cos \theta_{2} .
\end{gathered}
$$

To satisfy (3.1) we write the velocity potential as

$$
\phi=\sum_{m=0}^{\infty}\left[\alpha_{m}\left(\phi_{m}^{a(1)}-\phi_{m}^{a(2)}\right)+\beta_{m}\left(\phi_{m}^{s(1)}+\phi_{m}^{s(2)}\right)\right],
$$

where $\phi_{m}^{s(q)}$ and $\phi_{m}^{a(q)}$ are multipoles singular at $r_{q}=0$, respectively even and odd in $\theta_{q}$. The only condition remaining to be satisfied is then $\partial \phi / \partial r_{2}=0$ on $r_{2}=a$.

The zeros of transmission in the oblique wave scattering problem were found just below the cut-off frequency so we restrict attention to $K<l<k$ to obtain embedded trapped modes. In this region waves propagating to $|x|=\infty$ can exist with 
wavenumber $k$ (associated with the interface) but not with wavenumber $K$ (associated with the free surface).

To apply the body boundary condition, and hence solve for $\alpha_{m}, \beta_{m}, m \geqslant 0$, we require all the multipoles to be expanded in the polar coordinates $\left(r_{2}, \theta_{2}\right)$. The multipoles centred about $(\xi, f)$ are easily expanded in these coordinates and are given by

$$
\begin{aligned}
\phi_{m}^{s(2)} & =K_{m}\left(l r_{2}\right) \cos m \theta_{2}+\sum_{n=0}^{\infty} A_{m n}^{s} I_{n}\left(l r_{2}\right) \cos n \theta_{2}, \\
\phi_{m}^{a(2)} & =K_{m}\left(l r_{2}\right) \sin m \theta_{2}+\sum_{n=0}^{\infty} A_{m n}^{a} I_{n}\left(l r_{2}\right) \sin n \theta_{2},
\end{aligned}
$$

where $A_{m n}^{s}$ is given by (2.15) and

$$
A_{m n}^{a}=2(-1)^{m+n} \int_{0}^{\infty} \sinh m u \sinh n u \mathrm{e}^{2 v f} C(v) \mathrm{d} u .
$$

Note that the function $C$ now has a singularity on the real axis at

$$
\gamma=\cosh ^{-1}(k / l)
$$

and the path of integration is indented beneath this pole. The functions $A_{m n}^{s}$ and $A_{m n}^{a}$, and all the other similar integrals defined below, are thus complex-valued.

We write the multipoles which are centred about $(-\xi, f)$ as

$$
\begin{aligned}
& \phi_{m}^{s(1)}=K_{m}\left(l r_{1}\right) \cos m \theta_{1}+\psi_{m}^{s(1)}, \\
& \phi_{m}^{a(1)}=K_{m}\left(l r_{1}\right) \sin m \theta_{1}+\psi_{m}^{a(1)},
\end{aligned}
$$

where

$$
\begin{aligned}
\psi_{m}^{s(1)} & =(-1)^{m} \int_{0}^{\infty} \cosh m u \cos [l(x+\xi) \sinh u] \mathrm{e}^{v(z+f)} C(v) \mathrm{d} u \\
\psi_{m}^{a(1)} & =(-1)^{m+1} \int_{0}^{\infty} \sinh m u \sin [l(x+\xi) \sinh u] \mathrm{e}^{v(z+f)} C(v) \mathrm{d} u
\end{aligned}
$$

and $C(v)$ is defined in (2.16). We can expand $\psi_{m}^{s(1)}$ and $\psi_{m}^{a(1)}$ in the polar coordinates $\left(r_{2}, \theta_{2}\right)$ to obtain

$$
\begin{aligned}
& \psi_{m}^{s(1)}=\sum_{n=0}^{\infty}\left(A_{m n}^{s(1)} \cos n \theta_{2}+B_{m n}^{s(1)} \sin n \theta_{2}\right) I_{n}\left(l r_{2}\right), \\
& \psi_{m}^{a(1)}=\sum_{n=0}^{\infty}\left(A_{m n}^{a(1)} \cos n \theta_{2}-B_{m n}^{a(1)} \sin n \theta_{2}\right) I_{n}\left(l r_{2}\right),
\end{aligned}
$$

where

$$
\begin{gathered}
A_{m n}^{s(1)}=(-1)^{m+n} \epsilon_{n} \int_{0}^{\infty} \cosh m u \cosh n u \mathrm{e}^{2 v f} \cos (2 l \xi \sinh u) C(u) \mathrm{d} u, \\
B_{m n}^{s(1)}=-A_{n m}^{a(1)}=(-1)^{m+n} \epsilon_{n} \int_{0}^{\infty} \cosh m u \sinh n u \mathrm{e}^{2 v f} \sin (2 l \xi \sinh u) C(u) \mathrm{d} u, \\
B_{m n}^{a(1)}=(-1)^{m+n+1} \epsilon_{n} \int_{0}^{\infty} \sinh m u \sinh n u \mathrm{e}^{2 v f} \cos (2 l \xi \sinh u) C(u) \mathrm{d} u
\end{gathered}
$$


If we now apply the body boundary condition on $r_{2}=a$, expand $K_{m}\left(l r_{1}\right) \cos m \theta_{1}$ and $K_{m}\left(l r_{1}\right) \sin m \theta_{1}$ in the polar coordinates $\left(r_{2}, \theta_{2}\right)$ with the aid of Graf's addition theorem, and use the orthogonality of the trigonometric functions we obtain two coupled infinite systems of equations for the unknowns $\alpha_{m}$ and $\beta_{m}, m \geqslant 0$. These are

$$
\begin{gathered}
\alpha_{s}-\frac{I_{s}^{\prime}(l a)}{K_{s}^{\prime}(l a)} \sum_{m=0}^{\infty}\left(P_{m s} \alpha_{m}+Q_{m s} \beta_{m}\right)=0, \\
\beta_{s}+\frac{I_{s}^{\prime}(l a)}{K_{s}^{\prime}(l a)} \sum_{m=0}^{\infty}\left(R_{m s} \alpha_{m}+T_{m s} \beta_{m}\right)=0,
\end{gathered}
$$

$s \geqslant 0$, where

$$
\begin{aligned}
P_{m s}= & \frac{\epsilon_{s}}{2}\left(K_{m-s}(2 l \xi)+(-1)^{m+1} K_{m+s}(2 l \xi)\right) \cos (m+s) \frac{\pi}{2} \\
& +(-1)^{m+s} \epsilon_{s} \int_{0}^{\infty} \sinh m u \sinh s u \mathrm{e}^{2 v f}(\cos (2 l \xi \sinh u)-1) C(u) \mathrm{d} u, \\
Q_{m s}= & -\frac{\epsilon_{s}}{2}\left(K_{m-s}(2 l \xi)+(-1)^{m} K_{m+s}(2 l \xi)\right) \sin (m+s) \frac{\pi}{2} \\
& +(-1)^{m+s} \epsilon_{s} \int_{0}^{\infty} \cosh m u \sinh s u \mathrm{e}^{2 v f} \sin (2 l \xi \sinh u) C(u) \mathrm{d} u, \\
R_{m s}= & \frac{\epsilon_{s}}{2}\left(K_{m-s}(2 l \xi)+(-1)^{m+1} K_{m+s}(2 l \xi)\right) \sin (m+s) \frac{\pi}{2} \\
& +(-1)^{m+s+1} \epsilon_{s} \int_{0}^{\infty} \sinh m u \cosh \sin \mathrm{e}^{2 v f} \sin (2 l \xi \sinh u) C(u) \mathrm{d} u, \\
T_{m s}= & \frac{\epsilon_{s}}{2}\left(K_{m-s}(2 l \xi)+(-1)^{m} K_{m+s}(2 l \xi)\right) \cos (m+s) \frac{\pi}{2} \\
+ & (-1)^{m+s} \epsilon_{s} \int_{0}^{\infty} \cosh m u \cosh s u \mathrm{e}^{2 v f}(\cos (2 l \xi \sinh u)+1) C(u) \mathrm{d} u .
\end{aligned}
$$

We can write these systems of equations in matrix form as follows

$$
\boldsymbol{A x}=\left(\begin{array}{cc}
\boldsymbol{P}-\boldsymbol{I} & \boldsymbol{Q} \\
\boldsymbol{R} & \boldsymbol{T}+\boldsymbol{I}
\end{array}\right)\left[\begin{array}{l}
\boldsymbol{\alpha} \\
\boldsymbol{\beta}
\end{array}\right]=0,
$$

where

$$
\left.\begin{array}{ll}
\boldsymbol{P}=\left[I_{s}^{\prime}(l a) P_{m s} / K_{s}^{\prime}(l a)\right], & \boldsymbol{Q}=\left[I_{s}^{\prime}(l a) Q_{m s} / K_{s}^{\prime}(l a)\right], \\
\boldsymbol{R}=\left[I_{s}^{\prime}(l a) R_{m s} / K_{s}^{\prime}(l a)\right], & \boldsymbol{T}=\left[I_{s}^{\prime}(l a) T_{m s} / K_{s}^{\prime}(l a)\right], \\
\boldsymbol{\alpha}=\left[\alpha_{s}\right], & \boldsymbol{\beta}=\left[\beta_{s}\right],
\end{array}\right\}
$$

for $s \geqslant 0$ and $m \geqslant 0$. For non-trivial solutions we require the determinant of the complex matrix $\boldsymbol{A}$ to be zero. To find these points we truncate the matrix $\boldsymbol{A}$ to a $2 N \times 2 N$ system and calculate the determinant. By fixing, say, $\xi$ and varying $K$ we can find where the real and imaginary parts vanish. We use a wide-spacing approximation to aid in locating the points at which both the real and imaginary parts vanish.

\section{$A$ wide-spacing approximation}

The wide-spacing approximation has been used many times before to provide both good evidence for the existence of trapped-mode phenomena and also accurate and 


$\begin{array}{ccc}n & K a=0.4481 & K a=1.0825 \\ 1 & 2.8186 & 1.2254 \\ 2 & 5.2826 & 2.2515 \\ 3 & 7.7466 & 3.2776 \\ 4 & 10.2107 & 4.3037 \\ 5 & 12.6747 & 5.3298 \\ 6 & 15.1387 & 6.3559 \\ 7 & 17.6028 & 7.3820\end{array}$

TABLE 1. Values of $\xi / a$ predicted by the wide-spacing approximation; $\rho=0.5, d / a=2, f / a=-1.1$ and $\alpha_{\text {inc }}=0.34$.

efficient methods for the computation of the frequencies at which they occur (see, for example, Linton \& Kuznetsov 1997). Consider a wave of wavenumber $k$ with angle $\alpha_{\text {inc }}$ to the positive $x$-axis of the form $\exp [\mathrm{i} b(x-\xi)], b=\sqrt{k^{2}-l^{2}}=k \cos \alpha_{\text {inc }}$, incident upon the right-hand cylinder in figure 12 from $x=-\infty$ and assume that the other cylinder does not affect the interaction between the wave and the cylinder. Then, as shown in LC, there are certain values of $K a$ at which zeros of transmission occur and the wave is totally reflected. At such a frequency the reflection coefficient can be written as $r=\exp (\mathrm{i} \chi)$ for some $\chi \in \mathbb{R}$. In order for this solution to be consistent with the presence of the other cylinder we must have

$$
\mathrm{e}^{\mathrm{i} b(x-\xi)}+r \mathrm{e}^{-\mathrm{i} b(x-\xi)}=A \cos b x
$$

where $A$ is some complex constant. By substituting the form for $r$ we find this is equivalent to the condition

$$
\mathrm{e}^{\mathrm{i}(x+2 b \xi)}=1 .
$$

It follows that the distance of the cylinder from the wall must be related to the frequency at which total reflection occurs through the equation

$$
b \xi=n \pi-\frac{1}{2} \chi
$$

for some integer $n$.

\section{Results}

The values of $K a$ at which zeros of the real and imaginary parts of the determinant occur are plotted in figure 13 for a range of values of $\xi / a$ using $N=8$, and $\rho=0.5$, $d / a=2, f / a=-1.1$ and $\alpha_{\text {inc }}=0.34$. Note that $\alpha_{\text {inc }}$ is such that there are no propagating free-surface waves for all $K a$. The solid lines represent zeros of the real part of the determinant and the dashed lines represent zeros of the imaginary part. The two sets of curves appear to touch and the numbers computed strongly suggest that this is the case but because of the nature of the contact (the lines do not cross) one cannot be sure. An embedded trapped mode will exist if the solid lines and dashed lines do actually touch.

For the values of the parameters used here we find two frequencies at which zeros of transmission occur for the scattering of oblique waves: $K a \approx 0.4481$ (as shown in figure 5 of LC) and $K a \approx 1.0825$. Using a wide-spacing approximation we obtain estimates for the separation variable $\xi / a$ as shown in table 1 . The first column of $K a$ values corresponds to predicted values of $\xi / a$ in figure 13(b) whereas the final column corresponds to figure $13(a)$. It would appear that for $K a \approx 0.4481$ these are 

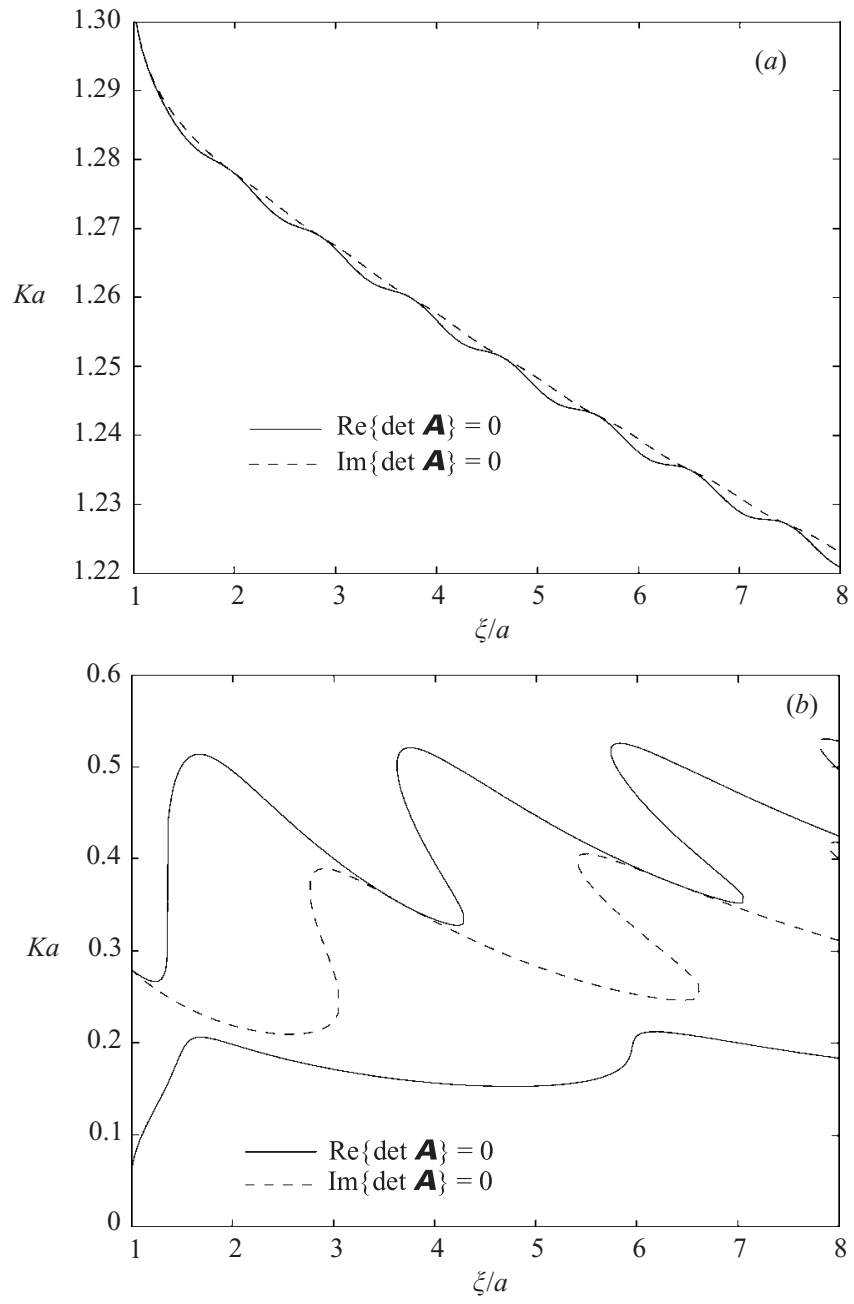

FIGURE 13. Values of $K a$ at which the real part (solid lines) and the imaginary part (dashed lines) of the determinant vanish; $d / a=2, f / a=-1.1, \rho=0.5$ and $\alpha_{\text {inc }}=0.34$.

poor estimates of where the curves possibly touch but for greater values of $\xi / a$ we find them more satisfactory, as might be expected.

It is clear from figure 13 that if we vary $\xi / a$ an arbitrarily small amount from the point of contact then the embedded trapped mode will cease to exist. These modes are unstable in the sense that a small change in the geometry results in the loss of the trapped mode. We now consider a different method which helps to establish that the curves do indeed touch.

\section{Reformulation of the problem}

We now consider the results if the complex-valued multipoles in (3.4) are replaced by real-valued multipoles in which the contour integrals are replaced by principal-value integrals. If we follow the above analysis with these real-valued multipoles (which behave like standing waves in the far field) the matrix $\boldsymbol{A}$ defined by (3.24) will be real and we will denote this real matrix by $\tilde{\boldsymbol{A}}$. The solutions of the reformulated problem, 


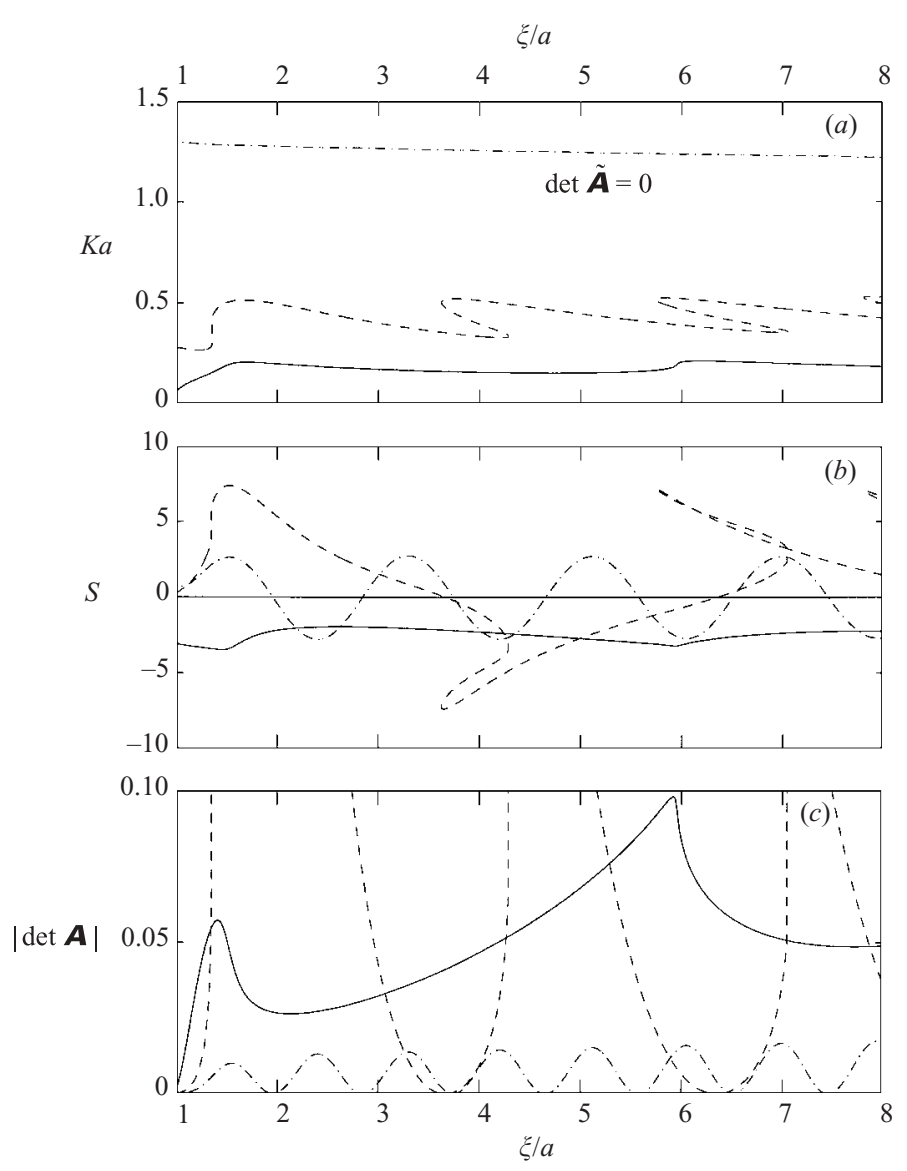

Figure 14. (a) Values of $K a$ at which the determinant of the real matrix vanishes with corresponding far-field sum $(b)$ and modulus of the determinant of the complex matrix $(c)$; $d / a=2, f / a=-1.1, \rho=0.5$ and $\alpha_{\text {inc }}=0.34$.

when $\operatorname{det}(\tilde{\boldsymbol{A}})=0$, will correspond to either trapped modes or standing waves in the entire fluid. This method was used by Evans \& Porter (1998) for the case of a rigid vertical circular cylinder placed on the centre-plane of a channel. To find the trapped-mode frequencies we must calculate the potential in the far field for each solution and find when it vanishes. As $x \rightarrow \pm \infty$, the symmetric multipoles satisfy

$$
\begin{aligned}
& \phi_{m}^{s(1)} \sim \mp(-1)^{m} C^{\gamma} \pi \cosh m \gamma \sin b(x+\xi) \mathrm{e}^{k(z+2 f)}, \\
& \phi_{m}^{s(2)} \sim \mp(-1)^{m} C^{\gamma} \pi \cosh m \gamma \sin b(x-\xi) \mathrm{e}^{k(z+2 f)},
\end{aligned}
$$

where

$$
C^{\gamma}=\frac{(k+K)\left[(k+K \sigma) \mathrm{e}^{-2 k d}-k+K\right]}{b(k-K)\left[(1-2 d(k+K)) \mathrm{e}^{-2 k d}-1\right]},
$$

and for the antisymmetric multipoles we simply replace cosh by sinh and sin by cos. To satisfy the far-field condition for embedded trapped modes we thus require, from (3.4), 


$$
S=\sum_{m=0}^{\infty}(-1)^{m}\left[\alpha_{m} \sinh m \gamma \sin b \xi-\beta_{m} \cosh m \gamma \cos b \xi\right]=0 .
$$

Hence we need to find the frequencies at which both the determinant of the new matrix $\tilde{\boldsymbol{A}}$ and also the summation in (3.32) vanishes. The coefficients $\alpha_{m}$ and $\beta_{m}$ in the sum are the eigenvectors of the matrix $\tilde{\boldsymbol{A}}$ corresponding to the eigenvalue zero. As an aside, because of the particular form of the elements of $\boldsymbol{A}$, it turns out that $\operatorname{Re}(\operatorname{det} \boldsymbol{A})=0$ if and only if $\operatorname{det} \tilde{\boldsymbol{A}}=0$.

Figure 14 shows results obtained from using the real-valued multipoles for the same values of the parameters as above: $f / a=-1.1, d / a=2, \rho=0.5, \alpha_{\text {inc }}=0.34$ and $N=8$. Figure $14(a)$ shows the values of $K a$ at which the determinant of the real matrix $\tilde{\boldsymbol{A}}$ vanishes as a function of $\xi / a$. Each curve has been given a different line style so that it can be recognized in the other plots. Figure $14(b)$ shows the corresponding far-field sums, $S$, and when they cross zero we have an embedded trapped mode. The points where the dashed curve crosses zero correspond to the approximations in the middle column of table 1 , while those for the dot-dashed curve correspond to the numbers in the right-hand column. The solid curve has no $S=0$ crossings and so never describes an embedded trapped mode. Figure 14(c) shows the modulus of the complex matrix $\boldsymbol{A}$ determinant where the frequency, $K a$, and separation, $\xi / a$, of the cylinder are given by the top graph. We can see that where there are zero crossings of the far-field sum the modulus of the complex matrix determinant touches zero. These figures provide strong evidence that the curves in figure 13 do indeed touch and thus that embedded trapped modes do exist for the geometry.

\section{Conclusion}

We have investigated trapped modes above a horizontal cylinder in a two-layer fluid consisting of a layer of finite depth on top of an infinitely deep layer of greater density. The trapped-mode frequencies for a cylinder situated in either the upper or lower layer were evaluated. For a cylinder in the lower fluid layer we found that the motion of the trapped modes was confined to the interface. When the cylinder is submerged entirely within the upper layer the trapped modes can be on either the free surface or the interface. By varying the density ratio of the fluid layers we found that a mode on the free surface can transfer to the interface and vice versa. For some density ratios it is possible to have trapped waves on the free surface and interface simultaneously with the same amplitude.

By letting the density ratio tend to zero, the two-layer fluid problems were related to problems in single-layer fluids. In this limit the trapped-mode problem in a two-layer fluid reduces to an infinite- or finite-depth single-layer fluid problem, depending on whether the cylinder is in the lower or upper layer. We also investigated the trapped modes as the density ratio tends to 1 and explained why the results do not go over to those for a single-layer infinite-depth fluid.

We have also considered the case of embedded trapped modes for the case of a pair of horizontal circular cylinders in a two-layer fluid. It is much more difficult to establish existence for embedded modes, but we have provided strong numerical evidence that trapped modes do exist above the cut-off for oblique waves for such a geometry. For the parameter values chosen, nine trapped modes embedded in the continuous spectrum have been found for nine separation values. The wide-spacing approximation suggests that there are an infinite number of embedded trapped modes 
corresponding to different distances between the cylinders, all other geometrical parameters being kept fixed.

\section{REFERENCES}

Berry, M. V. \& Wilkinson, M. 1984 Diabolical points in the spectra of triangles. Proc. R. Soc. Lond. A 392, 15-43.

Evans, D. V. \& Porter, R. 1998 Trapped modes embedded in the continuous spectrum. Q. J. Mech. Appl. Maths 51, 263-274.

JONES, D. S. 1953 The eigenvalues of $\nabla^{2} u+\lambda u=0$ when the boundary conditions are given on semi-infinite domains. Proc. Camb. Phil. Soc. 49, 668-684.

KuZnetsov, N. 1993 Trapping modes of internal waves in a channel spanned by a submerged cylinder. J. Fluid Mech. 254, 113-126.

Kuznetsov, N., Porter, R., Evans, D. V. \& Simon, M. J. 1998 Uniqueness and trapped modes for surface-piercing cylinders in oblique waves. J. Fluid Mech. 365, 351-368.

Kuznetsov, N., McIver, P. \& Linton, C. M. 2001 On uniqueness and trapped modes in the water-wave problem for vertical barriers. Wave Motion 33, 283-307.

Linton, C. M. \& CADby, J. R. 2002 Scattering of oblique waves in a two-layer fluid. J. Fluid Mech. 461, 343-364 (referred to herein as LC).

Linton, C. M. \& KuZnetsov, N. 1997 Non-uniqueness in two-dimensional water-wave problems: numerical evidence and geometrical restrictions. Proc. R. Soc. Lond. A 453, 2437-2460.

Linton, C. M. \& MCIver, M. 1995 The interaction of waves with horizontal cylinders in two-layer fluids. J. Fluid Mech. 304, 213-229.

Linton, C. M. \& MCIVER, P. 1998 Acoustic resonances in the presence of radial fins in circular cylindrical waveguides. Wave Motion 28, 99-117.

MCIver, M. 1996 An example of non-uniqueness in the two-dimensional linear water wave problem. J. Fluid Mech. 315, 257-266.

McIver, M. 2000 Trapped modes supported by submerged obstacles. Proc. R. Soc. Lond. A 456, $1851-1860$.

McIver, P. \& Evans, D. V. 1985 The trapping of surface waves above a submerged horizontal cylinder. J. Fluid Mech. 151, 243-255.

Motygin, O. V. 1999 Trapped modes of oscillation of a liquid for surface-piercing bodies in oblique waves. J. Appl. Math. Mech. 63, 257-264.

Porter, R. 2002 Trapping of water waves by pairs of submerged cylinders. Proc. R. Soc. Lond. A 458, 607-624.

Ursell, F. 1951 Trapping modes in the theory of surface waves. Proc. Camb. Phil. Soc. 47, 347-358. 